\title{
Otitis externa por Staphylococcus aureus resistente a meticilina en un lactante
}

\author{
P. Gorrotxategi Gorrotxategia ,JM. Manterola Martija ${ }^{\mathrm{b}}$ \\ aPediatra. CS Ermua. Bizkaia. España.

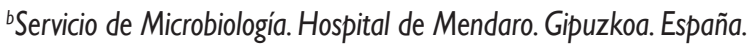

\begin{abstract}
Resumen
Se presenta el caso clínico de un niño que presentó una otitis externa a los dos meses de edad. El cultivo ótico mostró un Staphylococcus aureus resistente a meticilina (SARM). Se realizó un estudio familiar en ambos padres resultando los cultivos nasales negativos. Posteriormente, estudiando la historia familiar se supo que la madre había tenido en el momento del parto un cultivo de herida quirúrgica positivo a SARM con el mismo antibiograma que el que presentaba el niño. Como en ese periodo de tiempo no se había hecho ningún tratamiento en el niño se volvió a repetir el cultivo para valorar la necesidad o no de tratamiento persistiendo el SARM. Se realizó tratamiento local con ciprofloxacino, siendo el cultivo de control negativo.
\end{abstract}

Palabras clave: Staphylococcus aureus resistente a meticilina. Otitis. Transmisión vertical. Niño.

External otitis by Methicillin-resistant Staphylococcus aureus in an infant

Abstract

We reported the case of a child two months old, with external otitis by Methicillin-Resistant Staphylococcus Aureus (MRSA). Nasal swabs were collected from the father and the mother with negative result. An intra-familial vertical transmission was identified later.

Key words: Methicillin-resistant Staphylococcus aureus. Otitis. Vertical transmission. Child.

\section{Introducción}

Staphylococcus aureus es una de las principales causas de infecciones en humanos, tanto en el medio hospitalario como en la comunidad. Son responsables principalmente de infecciones de piel y partes blandas, bacteriemia, endo- carditis y neumonía, pero además producen un creciente número de infecciones relacionadas con la utilización de diferentes tipos de catéteres y otros dispositivos médicos. Asimismo, los estafilococos poseen una extraordinaria capacidad para desarrollar resistencia a

Pedro Gorrotxategi Gorrotxategi, pedrojesus.gorrotxategigorrotxategi@osakidetza.net

Los autores declaran no presentar conflictos de intereses en relación con la preparación y publicación de este artículo.

El trabajo ha sido presentado como comunicación póster a la 6 . $^{a}$ reunión de la Asociación Española de Pediatría de Atención Primaria en Valencia en 2010. 
antimicrobianos. En los últimos años se ha observado un incremento y la diseminación de cepas de $S$. aureus resistentes a meticilina (SARM) tanto en el medio sanitario como en otras cepas adquiridas en la comunidad.

Según los datos del Grupo Español para el Estudio de Estafilococo ${ }^{1}$, los aislamientos de SARM se han mantenido prácticamente estables desde el año 2002 (31,2\%) a 2006 (29,4\%). En cuanto a la presencia de gérmenes aislados de SARM a partir de muestras del medio extrahospitalario, las cifras encontradas demuestran también la estabilidad en estos últimos años (el $17,8 \%$ en 2002 , frente al $17,9 \%$ en 2006). Similares incidencias encontramos en el estudio colaborativo EARSS ${ }^{2}$ (European Antimicrobial Resistance Surveillance System). Según este estudio el $24,5 \%$ de los $S$. aureus aislados en sangre eran resistentes a la oxacilina y de estos un $68,1 \%$ eran multirresistentes.

Pero si esos porcentajes correspondían a cultivos de sangre $u$ otras localizaciones, un dato que nos permite conocer la verdadera extensión de este germen es la colonización nasal del mismo. Datos provenientes de EE. UU. reportan un aumento de los cultivos nasales con SARM adquiridos en la comunidad ${ }^{3}$. Según el estudio citado, la incidencia poblacional ha pasado de un 1 a un 9,2\%, entre 2001 y 2004, incluyendo tanto a niños como a adultos. Otro estudio que analiza los cultivos nasales exclusivamente en niños obtiene una cifra de prevalencia de SARM del 2,6\%, de los cuales el $28 \%$ está relacionado con la asistencia sanitaria y el $66 \%$ adquirido en la comunidad ${ }^{4}$. Dado que la colonización nasal precede a la infección, los autores expresan su temor de que las infecciones por SARM aumenten en los próximos años.

Presentamos el caso clínico de un lactante con una otitis externa (contaminación del conducto auditivo externo por un SARM) por la rareza del hallazgo y como motivo de reflexión sobre el aumento de este germen y sobre la importancia de la recogida de muestras microbiológicas para el adecuado seguimiento de nuestros pacientes.

\section{Caso clínico}

Lactante de un mes y 20 días, hijo único, que acude a la consulta de Atención Primaria por presentar una secreción blanquecina en conducto auditivo externo (CAE).

Se realiza un cultivo de dicha secreción, que resulta positivo para SAMR (tabla 1). Se realiza un estudio familiar, obteniéndose cultivo nasal en ambos padres, que resulta negativo. 


\begin{tabular}{llllll}
\hline Tabla 1. Antibiograma del cultivo ótico del niño y cultivo exudado herida materna & \\
\hline Antibiograma & \multicolumn{2}{c}{$\begin{array}{c}\text { Niño (CMI) } \\
(23-03-2009)\end{array}$} & $\begin{array}{c}\text { Madre } \\
(11-02-2009)\end{array}$ & $\begin{array}{c}\text { Niño (CMI) } \\
(08-04-2009)\end{array}$ \\
\hline Penicilina & Resistente & 8 & Resistente & Resistente & $\geq 0,5$ \\
\hline Oxacilina & Resistente & $\geq 4$ & Resistente & Resistente & $\geq 4$ \\
\hline Gentamicina & Sensible & $\leq 4$ & Sensible & Resistente & $\geq 16$ \\
\hline Tobramicina & - & - & - & Resistente & $\geq 16$ \\
\hline Eritromicina & Sensible & $\leq 0,25$ & Sensible & Resistente & $\geq 8$ \\
\hline Tetraciclina & Sensible & $\leq 2$ & Sensible & Resistente & $\geq 16$ \\
\hline Clindamicina & Sensible & 0,5 & Sensible & Resistente & $\geq 8$ \\
\hline Vancomicina & Sensible & $\leq 1$ & Sensible & Sensible & $\leq 1$ \\
\hline Ciprofloxacino & Resistente & $\geq 4$ & Resistente & Sensible & $\leq 0,5$ \\
\hline Trimetoprim/sulfametoxazol & Sensible & $\leq 2 / 38$ & Sensible & Resistente & $\geq 4 / 76$ \\
\hline Rifampicina & Sensible & $\leq 1$ & Sensible & Intermedio & 2 \\
\hline Telitromicina & - & - & - & Resistente & $\geq 4$ \\
\hline CMl: concentración mínima inhibitoria. & & & & \\
\hline
\end{tabular}

Al analizar la historia familiar, tenemos conocimiento de que la madre había presentado una herida quirúrgica en el momento del parto, cuyo cultivo fue positivo para SARM con el mismo antibiograma que el hallado en el niño en el CAE (tabla 1), por lo que suponemos que se ha producido una transmisión vertical de dicho germen. Tras realizar estas investigaciones, se vuelve a tomar una muestra del CAE del niño, obteniéndose siendo de nuevo positivo para SARM, cuyo antibiograma se muestra en la tabla 1.
Dado que este segundo cultivo es sensible a ciprofloxacino, se realizó tratamiento local con esa quinolona siendo el cultivo de control negativo.

\section{Discusión}

Tras conocer que el niño tenía una otitis externa por SARM, se puso en marcha el estudio para descartar que fuera un SARM de origen comunitario, que como hemos relatado es lo más frecuente en niños, las $2 / 3$ partes según un estudio poblacional ${ }^{4}$. Para pensar que el SARM 
ha sido adquirido en la comunidad no hay que tener en cuenta factores de riesgo especiales. Según un estudio realizado en el Hospital Central de Taiwán, dentro de los SARM de origen comunitario no siempre se encuentran factores de riesgo concretos; así, el 9,8\% de los aislamientos de SARM se dió en pacientes sin factores de riesgo; el 17,9\%, en los que tenían factores de riesgo, y el $72,3 \%$ fueron $S$. aureus sensibles a meticilina ${ }^{5}$.

Se realizó, por lo tanto, un examen del cultivo nasal. Según las descripciones de la literatura médica, para el estudio del estado de portador se suele realizar a los pacientes y a sus familiares cultivos de frotis nasal y de los pliegues axilar e inguinal. Mediante esta técnica se obtiene un resultado positivo en la mayoría de los casos. En una serie de diez casos recientemente publicada se pudo realizar el estudio del estado de portador, que fue positivo en seis de ellos. Se aisló SARM adquirido en la comunidad en 12 muestras (ocho frotis nasales, tres frotis cutáneos y un exudado conjuntival) $)^{6}$. En dichos casos, la mayoría de las infecciones fueron cutáneas: impétigo con celulitis y abscesos subcutáneos, fascitis necrosante y onfalitis, aunque también hubo abscesos profundos y algún caso de osteomielitis. En cuanto al origen, la mitad eran emigrantes (dos de Rumanía y cuatro de Ecuador).
Tras descartar la presencia de un SARM de origen comunitario contagiado por los padres, al resultar ambos cultivos paternos negativos, como ya hemos relatado, al repasar la historia materna se observó que en ella constaba un cultivo vaginal positivo para SARM con el mismo espectro de sensibilidad antibiótica que el cultivo ótico del niño. La investigación de SARM en cultivos vaginales, previos al parto, se ha realizado en algún estudio con la finalidad de prevenir la infección invasiva de comienzo precoz en el recién nacido. La colonización materna por $S$. aureus se daba en el $14,5 \%$ de las mujeres y un $24,3 \%$ de ellos eran SARM, lo que nos indica que la prevalencia de SARM en los cultivos vaginales de mujeres embarazadas es del 3,5\%7. Otros estudios ofrecen resultados similares, es decir, un $17 \%$ de colonización por $S$. aureus y un $2,8 \%$ de $\mathrm{SARM}^{8}$. Un aspecto en el que coinciden ambos estudios es la asociación existente entre cultivo genital positivo a Streptococcus del grupo B (SGB) y la presencia tanto de $S$. aureus como de SARM. En el primero de los estudios citados, las mujeres SGB positivas tuvieron un probabilidad significativamente mayor que las SGB negativas tanto para la infección por S.aureus (20,7\% frente a $12,7 \%$ ) como en la presencia de SARM (6\% frente a $2,8 \%)^{7}$. A pesar de 
que ninguno de los dos estudios recomienda una detección sistemática de $S$. aureus, sí indican que debe existir una vigilancia de este germen para conocer su evolución, sobre todo en los casos SGB positivos.

Como consideración final, el SAMR, además de enfermedad invasiva, como neumonía osteomielitis, sepsis precoz del recién nacido o bacteriemia, entidades que son bastante raras, con mucha mayor frecuencia puede dar lugar a contaminación cutánea superficial y en algu- nos casos, como el presentado, afectar al CAE. En un recién nacido con infección cutánea por SARM, uno de los focos de infección que debemos investigar es la genital de su madre, para descartar una transmisión vertical del mismo.

Por otro lado, este caso nos enseña también la conveniencia de realizar toma de cultivos de las secreciones desde Atención Primaria, sea de la localización que sea, ya que la información obtenida nos ayuda al correcto tratamiento y seguimiento de nuestros pacientes.

\section{Bibliografía}

1. Cuevas $O$, Cercenado E, Goyanes MJ, Vindel A, Trincado P, Boquete T y cols.; Grupo Español para el Estudio de Estafilococo. Staphylococcus spp. en España: situación actual y evolución de la resistencia a antimicrobianos (1986-2006). Enferm Infecc Microbiol Clin. 2008;26:269-77.

2. Oteo J, Baquero F, Vindel A, Campos J; Spanish members of the European Antimicrobial Resistance Surveillance System. Antibiotic resistance in 3113 blood isolates of Staphylococcus aureus in 40 Spanish hospitals participating in the European Antimicrobial Resistance Surveillance System (2000-2002). J Antimicrob Chemother. 2004;53: 1033-8.

3. Creech CB, Kernodle DS, Alsentzer A, Wilson C, Edwards KM. Increasing rates of nasal carriage of methicillin-resistant Staphylococcus aureus in healthy children. Pediatr Infect Dis J. 2005;24:617-21.
4. Firtz SA, Garbutt J, Elward A, Shannon W, Storch GA. Prevalencia y factores de riesgo de colonización por Stapylococcus aureus resistente y sensible a meticilina adquirido en la comunidad en niños visitados en una consulta de pediatría afiliada a una red de investigación basada en consultorios. Pediatrics (Ed esp). 2008;65:291-9.

5. Wu KC, Chiu HH, Wang JH, Lee NS, Lin HC, Hsieh CC et al. Characteristics of communityacquired methicillin-resistant Staphylococcus aureus in infants and children without known risk factors. J Microbiol Immunol Infect. 2002;35(1): 53-6.

6. Frick MA, Moraga-Llop FA, Bartolomé R, Larrosa $N$, Campins $M$, Roman $Y$ y cols. Infecciones por Staphylococcus aureus resistente a meticilina adquirido en la comunidad en niños. Enferm Infecc Microbiol Clin. 2010;28:675-9.

7. Andrews WW, Schelonka R, Waites K, Stamm A, Cliver SP, Moser S. Genital tract methi- 
cillin-resistant Staphylococcus aureus: risk of vertical transmission in pregnant women. Obstet Gynecol. 2008;111(1):113-8.
8. Chen KT, Huard RC, Della-Latta P, Saiman L. Prevalence of methicillin-sensitive and methicillinresistant Staphylococcus aureus in pregnant women. Obstet Gynecol. 2006;108:482-7. 